DOI: 10.30519/ahtr.537204

Advances in Hospitality and Tourism Research (AHTR)

\title{
THE PARADOX OF CRACKER BARREL: A CASE STUDY ON PLACE AND PLACELESSNESS
}

\author{
Meredith GREGORY \\ University of Mary Washington, Virginia, USA \\ ORCID: 0000-0002-0223-8448 \\ Caitlin FINLAYSON ${ }^{1}$ \\ University of Mary Washington, Virginia, USA \\ ORCID: 0000-0003-3611-9970
}

\begin{abstract}
Cracker Barrel restaurants are a fixture across the interstate landscape of the United States. These sites cultivate a strong sense of place through careful theming, generating a distinct sense of rural America and nostalgia for home. At the same time, the uniformity of Cracker Barrel speaks to the notion of placelessness, the eradication of unique local features and homogenization of experience. Cracker Barrel is thus simultaneously placeless and placefull. This research explores this paradoxical notion by utilizing semiotic analyses in an analysis of user-generated Yelp! photos of Cracker Barrels across the country. It is clear from this analysis that the number and
\end{abstract}

Article History

Received 8 March 2019

Revised 29 October 2019

Accepted 1 November 2019

Keywords

theming

restaurants

place making

\section{INTRODUCTION}

Cracker Barrel restaurants are beacons of home-cooking along interstates across the United States and welcome 230 million guests yearly to their reproduction of a small, rural town's general store from early America in the 1900s. In hopes of providing a consistent, home-away-from-home,

1 Address correspondence to Caitlin Finlayson, University of Mary Washington, 1301 College Avenue Fredericksburg, Virginia, USA. E-mail: cfinlay@umw.edu 
Cracker Barrel carefully designs the decor, the food, and the atmosphere to align with the rural general store theme at each of their 658 locations.

Cracker Barrel works diligently to create a strong sense of place in their restaurants yet simultaneously exists as a placeless place. This sense of place, however, is contrasted with the inescapable placelessness, or lack of unique, distinguishing features (Relph, 1976), that is a result of all 650 Cracker Barrels looking virtually the same. The focus of this research is on how Cracker Barrel intentionally works to create a sense of place, while at the same time, how its uniformity simultaneously positions it as a placeless place. Cracker Barrels are designed and decorated the same no matter where they are geographically located leading them to be considered placeless. Even with minimal ties to the local area, there are few places, restaurant chains especially, that foster a greater sense of place for so many people. Through placemaking and theming - "the process by which an environment is given a distinct character" (Muñoz \& Wood, 2009, p. 270) - Cracker Barrel effectively reproduces the feeling of a small, rural town's general store from the early 1900s for all the patrons to enjoy. Thus exploring how this sense of place is created, and recreated on a vast scale, is important to explore. For Cracker Barrel in particular, there is an appeal to both tourists and locals alike and thus a paradox between maintaining uniqueness amidst a wide geographic area and differentiation among a busy restaurant landscape.

This paper uses a visual, semiologic methodology to explore the paradox of placelessness and placefullness at Cracker Barrel restaurants. By using user generated Yelp! photos from a stratified random sample of stores across the country, this study analyzes how Cracker Barrel's design choices create a unique sense of place while also proving the hypothesis that the stores do not change with the changing geographies. Ultimately, the results of this research provide an understanding of how Cracker Barrel uses symbols to create place, which elements vary across the country and which do not, as well as a discussion on Cracker Barrel's motivations.

\section{PLACE, PLACELESSNESS, AND THEMING IN CRACKER BARREL RESTAURANTS}

Cracker Barrel's defining feature is its distinctive place-making, creating a palpable sense of home through its nostalgic decor and menu items. The notion of place more broadly is a defining feature of geographic study 
(Nelson, 2017) and is highly emotional. As Tuan (1977, p. 6) notes "what begins as undifferentiated space becomes place as we get to know it better and endow it with value". Placemakers, such as those designing Cracker Barrels, rely on the ability to create place by changing the physical characteristics but can only hope their designs create an atmosphere where patrons become attached and create their own personal sense of place in the restaurants.

Place attachment refers to the ability of a place to make someone feel a certain way. Shao and Lui $(2017$, p. 152) define place attachment as "an effective bond that people establish with specific areas where they prefer to remain and where they feel comfortable and safe". The key, therefore, to place attachment is that people become attached to familiar places whether that be from length of residency, bonds with neighbors, or other factors (Phillips et al., 2011, p. 78). Place attachment is a continual and never ceasing process (Cuba \& Hummon, 1993, p. 547), yet the time it takes to truly become attached to one place in particular is rarely immediate and takes a long time to fully develop (Phillips et al., 2011, p. 77). Tuan (1977, p. 184) furthers this idea stating, "attachment, whether to a person or to a locality, is seldom acquired in passing". Just as people form relationships with other people, so too can they connect with places.

Various symbols anchor a person's sense of place, and collectively, these objects serve as place-makers for the community (Phillips et al., 2011, p. 87). Csurgó and Megyesi (2016, p. 430) highlight the importance of this stating, "symbolization of place is one of the main dimensions of place making". Not only do objects create a sense of place, but they also "anchor time," as Tuan (1977) writes. Place designers connect with these ideas in designing themed restaurants, historic sites, and some personal homes. These locations may therefore establish themselves as historic deliberately through the use of artifacts (Tuan, 1977, p. 198). Two broad categories used by place designers are domestication, or the production of familiar landscape, and exoticisation, or the formation of unfamiliar landscapes (Korusiewicz, 2015, p. 401). Typically, people get attached to familiar areas as they "find comfort in familiarity" (Phillips et al., 2011, p. 78). Korusiewicz (2015, p. 401), however, comments on the "fascinating mystery" experienced in exotic places that draws people to the place allowing them to form attachments.

While certainly placefull, Cracker Barrel is also placeless as a Cracker Barrel in North Carolina looks and feels the same as a Cracker Barrel in Arizona or anywhere else in the country. Relph (1976) defines 
placelessness as "the casual eradication of distinctive places and the making of standardized landscapes" (Relph, 1976, Preface). Harner and Kinder (2011, p. 751) argue that the physically distinct attributes of a place allow for place attachments by individuals and communities, and thus the elimination of those attributes results in a placeless place. Korusiewicz (2015, p. 402) similarly defines placelessness as "the lack of a recognizable focal point (or points) of mental and material space that one could connect to". It is this lack of unique features that leads to landscapes "looking more and more like everywhere else" (Harner \& Kinder, 2011, p. 752).

Phillips et al. (2011, p. 81), however, challenge this definition due to the lack of concern for the temporal, social, or individual meaning that create places. They define placelessness as "a lack of 'insideness' and meaning, anomie or simply not knowing a place" (Phillips et al., 2011, p. 81). They continue by attributing this to potential social exclusions or fleeting experiences with a place in which there is not enough time to gain a sense of place. When reflecting back on the previous discussion of place attachment, a short amount of time in a place severely limits the likelihood of feelings of social insideness or autobiographical attachment leaving only the physical characteristics of a place to become attached to.

No matter what the leading cause of placelessness, the result is always the same: a "sterile" (Korusiewicz, 2015, p. 402), "cookie-cutter" (Hough, 1990, p. 183), "standardized" (Relph, 1976, Preface), "anchorless" (Goss, 1992, p. 172), "homogenized blandscape" (Relph, 1981, p. 13; Phillips et al., 2011, p. 83; Zelinsky, 2011, Preface). Zelinsky (2011) opens his book commenting on the current state of the American population and landscape. He writes, "we are a population, after all, that spends much of its time outside the home (all too often a cookie-cutter structure devoid of regional resonance, where television mesmerizes us several hours of the day) shopping or eating in look-a-like chain or franchise operations, driving along featureless highways built into governmental specifications, sitting in anonymous airports, and sleeping in forgettable motels" (Zelinsky, 2011, p. 1). Zelinsky (2011) stresses the lack of connection between these features and the place where they are located (Zelinsky, 2011, p. 1). Not only are the elements listed above divorced from place, but they are also stripped of the uniqueness that defines places (Zimmerbauer, 2011 , p. 247). Phillips et al. (2011, p. 83) further highlights the shift from distinctive shops and landmarks to "homogenized" features that dominate much of the United States. 
Themes are socially constructed elements of the built environment that represent a specific time, place, or culture (Wood \& Muñoz, 2007, p. 243). Wood and Muñoz (2007, p. 243) centralize theming to the study of place when they state, "we live in a themed world" be it malls, museums, retailers, amusement parks, events, or restaurants. Muñoz and Wood (2009) argue the most commonly experienced of these is restaurants. The time periods, places, and cultures mimicked are themselves socially constructed as people over time decided how these were each defined (DeLyser, 1999, p. 606; Wood \& Muñoz, 2007, p. 244). Themed worlds further act as surrogates for the actual environment they represent (Wood \& Muñoz, 2007, p. 243). Wood and Muñoz (2007, p. 243) list ethnic art, decor, music, external façades, the name, among other stereotypical features as elements that create these themes. These function as the symbols that both create and reproduce a sense of place, a sense of culture, and/or a sense of time (Zimmerbauer, 2011, p. 246). In the case of restaurants, the food is also a symbol of the host culture, place, or time (Wood \& Muñoz, 2007, p. 243).

Themed restaurants use stereotypical and expected symbols that have been learned and practiced over time to create an experience that is standardized (Wood \& Muñoz, 2007, p. 243). It is from these standardized experiences that the public visiting these sites gets a sense of culture potentially within a very different culture meaning the patron may not get the true, authentic experience of that place (Wood \& Muñoz, 2007, p. 243). While many patrons visit Outback Steakhouse, to borrow Muñoz and Wood's (2009) example, and understand it as an "idealized and orchestrated portrayal" of Australia, there are others that see Outback as a truly authentic and real representation of Australia (Wood \& Muñoz, 2007, p. 243). The theming of restaurants is "significantly visual" as the businesses' "reality engineers" strategically construct environments that evoke certain feelings from the patrons (Wood \& Muñoz, 2007, p. 243; Muñoz \& Wood, 2009, p. 270). Muñoz and Wood (2009) continue stating, "when selecting design atmospheric and aesthetic cues, reality engineers rely heavily on socially constructed, yet often inaccurate themes offered by destination image formation agents" (Muñoz \& Wood, 2009, p. 270).

Accurate or not, these places rely on objects to create the themes and thus the place. As placelessness is generally defined as a place lacking distinguishing features, the use of such standardized symbols to create themed areas, such as koalas and kangaroos in Australian themed restaurants, leads to themed establishments that, when looked at broadly, can be considered placeless. Assuming these areas are located outside the 
culture they represent, these places stand out from the surrounding restaurants and stores, which would allow them to be considered distinctively placefull, yet their homogenous presentation makes them also essentially placeless, a critical paradox.

\section{METHODS}

This research aims to increase understanding of how Cracker Barrel creates a sense of place while simultaneously existing as a placeless place through a visual, semiotic analysis of user-generated Yelp images, following the framework established by DeBres and Sowers (2009). Cracker Barrel Old Country Store is a unique, company-owned and operated restaurant chain with locations on interstates across the country that aim to be the patron's "home-away-from-home" ("Frequently Asked Questions," n.d). Each location features a large front porch with rocking chairs and checkers, a general store with hosts of old-fashioned candy and soda, homeware, and other products designed to take the visitor back in time, and a restaurant serving traditional Southern home-style meals and decorated floor to ceiling with antique artifacts (See Figure 1). The original Cracker Barrel still exists in Lebanon, TN and was established in 1969. As popularity grew the company expanded, primarily in the Southeast, but as of 2019 has 658 stores in 45 states.
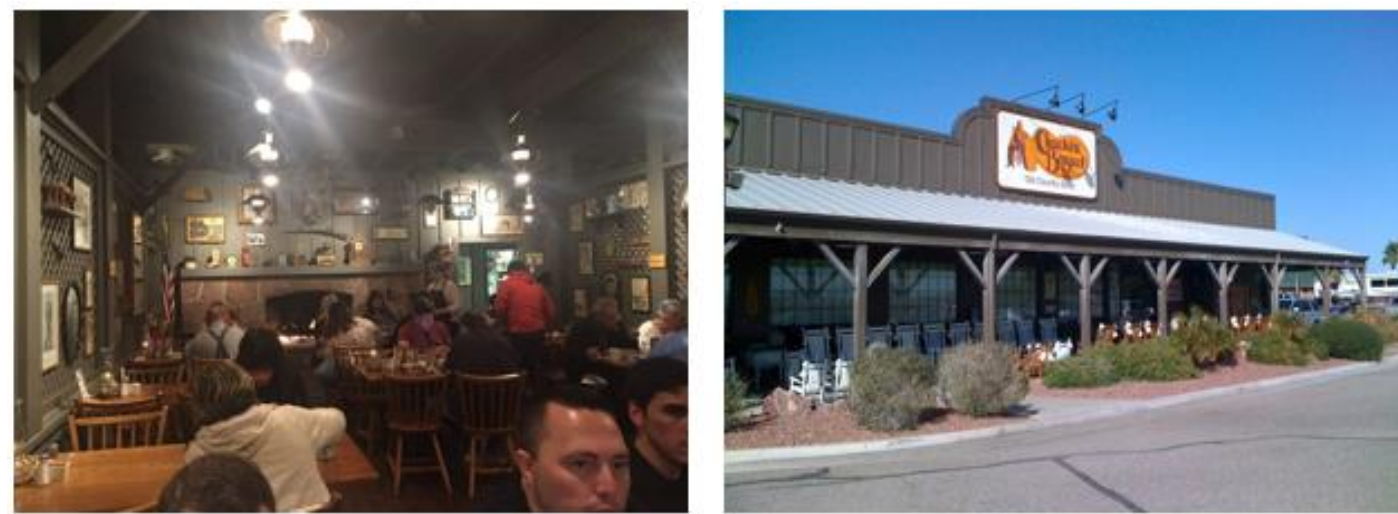

Yuma, Arizona

Figure 1. Yuma, Arizona Cracker Barrel²

\footnotetext{
2 The image on the left highlights the dining area of the Yuma, Arizona Cracker Barrel and the image of the right show the outside of the same store. Almost all Cracker Barrels look nearly identical to this location. Sources (left to right): L, C. Restaurant Overview. Yelp!, 29 Jan. 2016, s3-media4.fl.yelpcdn.com/bphoto/iC3hiDyV3Spf9VuDUPQMQ/o.jpg; C., Chris. Exterior. Yelp! 15 March 2010, s3media2.fl.yelpcdn.com/bphoto/UwTgrP4Xr-iwY9UCAk7iyw/o.jpg
} 
As a restaurant and store chain with a national presence, Cracker Barrel markets itself as a nostalgic experience of days gone by and as a home-away-from-home for weary, interstate travelers. In this way, Cracker Barrel stores present an intersection between placefulness, placelessness, and theming that provides a robust landscape for researchers to explore. Their goal of domestic placemaking, as defined above by Korusiewicz (2015), is however only realized by those visitors for whom this old timey country store culture is familiar whether that be because the store is located in an area where this culture presides or that these visitors stop in at a store elsewhere in the country.

This research utilizes a visual methodology examining usergenerated Yelp! photos and captions from Cracker Barrels across the country. The study's intent was to identify those elements that do not vary with geography as well as those that do. This research will also examine how these specific elements work together to create the placefullness found at the stores. The authors used a stratified random sample of locations, selecting one store at random from the 43 states where Cracker Barrel had a location at the time this research was carried out. After selecting the stores, we downloaded all of the Yelp! user-generated images for each store, excluding those images which depicted food since the menu is uniform from store to store. Yelp! provided one common place from which to view each store in the study as well as a customer perspective rather than company generated images. These images allowed the authors to view the Cracker Barrel experience from the customer perspective. Once all of the 746 images were downloaded and stored, they were coded and analyzed using Google Sheets.

Semiotic analysis, following the model of Rose (2016), was used to explore how Cracker Barrel attempts to create a sense of place while simultaneously existing as a placeless place by identifying and analyzing the signs in the restaurants that create both the placefullness and placelessness of Cracker Barrel. Semiotic analysis, put simply, is the "study of signs" (Crang, 2005; Rose, 2016) based upon the work by Ferdinand de Saussure. In semiotic studies such as this, "there is no concern... to find images that are statistically representative of a wider set of images... as there is in content analysis" (Rose 2016, p. 110). Rather, semiological investigations are often "detailed case studies of relatively few images" (Rose 2016, p. 110). Saussure (1998) explored how language is more than simply combinations of sounds and letters but represents deeper ideas of shared meaning. This conceptualization provides a 
foundation for visual studies of semiology, similarly examining how images can convey deeper "signified meanings" (Rose 2016, p. 114).

Semiotic analysis is an approach utilized in a number of disciplines, particularly tourism studies (see Echtner, 1999; Mehmetoglu \& Dann, 2002; Pennington \& Thomsen, 2010; Lau, 2011; Knudsen \& Rickly-Boyd, 2012; Lau, 2014; Song \& Jeon, 2018 and cultural studies (see Williamson, 1978). For geographers examining tourism and hospitality, a semiotic approach to visual analysis can provide exceptional insight into the impact of visual cues both in print media and in the environment around us. DeBres and Sowers (2009, p. 220), for example, used a combination of content and semiotic analysis to investigate "how denotative signs were manipulated to present a connotative sign to the viewer" in a study of 140 main street post cards. Applying their approach to this study, at Cracker Barrel, denotative signs are the artifacts on the walls and the old oil lamps on the tables while the connotative signs are the homeiness and time period that these artifacts work together to create. A key here is the interplay between the signs as "signs derive their meaning from other signs and from the wider system of signs" present (Crang, 2005, p. 277). A shovel, for example, means something different when in a tool shed than it does on the wall of a Cracker Barrel aside many other artifacts of rural 19th century America. DeBres and Sowers (2009) make the point as well that the meaning of connotative signs varies geographically as mentioned above but also by culture; they continue stating that "within a particular culture, [connotative signs] can be used repeatedly to sell and reaffirm a society's core myths and ideals" (DeBres \& Sowers, 2009, p. 220) which is just how Cracker Barrel uses them. The use of semiotic analysis, which provides a more in-depth look at the interaction of the specific signs, provides a useful approach to investigating the paradox of placefulness/placelessness in Cracker Barrel restaurants.

\section{RESULTS}

The deliberate use of artifacts creates a sense of place that make Cracker Barrel both placeless and placefull, and this is readily apparent through an analysis of customer images of these sites. The stores all varied in the number of images posted on Yelp! with some stores having over 80 pictures of the store, not including any pictures of food, and others having less than 10. Most images (50\%) depict the inside of Cracker Barrel's restaurant area, with others showing the outside areas $(26 \%)$ or the general store $(24 \%)$. Overall, the analysis of the images reveals both 
explicit and implicit details regarding how consumers experience, and document their experience, of Cracker Barrel locations.

When analyzing consumer images, it is clear that very little changes from one Cracker Barrel to the next (See Figure 2). Many of the stores and much of the design remains virtually unchanged, despite a few minor differences. Placelessness, as explained in the literature review, is the result of the "eradication of distinct landscapes" (Relph, 1976, Preface), the lack of connectedness to a place whether personally or physically (Harner \& Kinder, 2011, p. 751), and results in everywhere looking like everywhere else (Harner \& Kinder, 2011, p. 752). Cracker Barrels, while distinctly different than most of their surroundings, exist virtually unchanged place to place regardless of their geographic location.

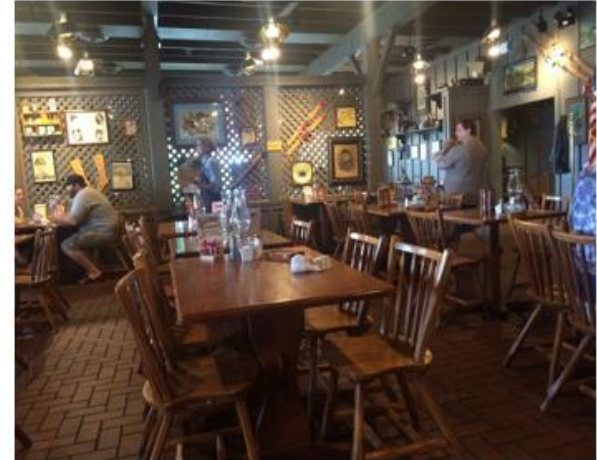

Milford, Connecticut

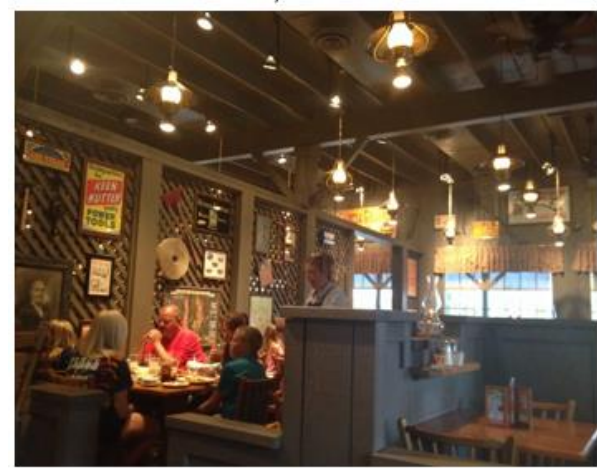

Stevensville, Maryland

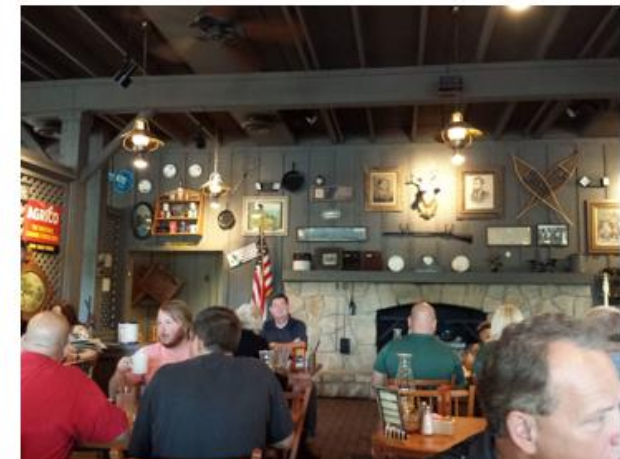

Okemos, Michigan

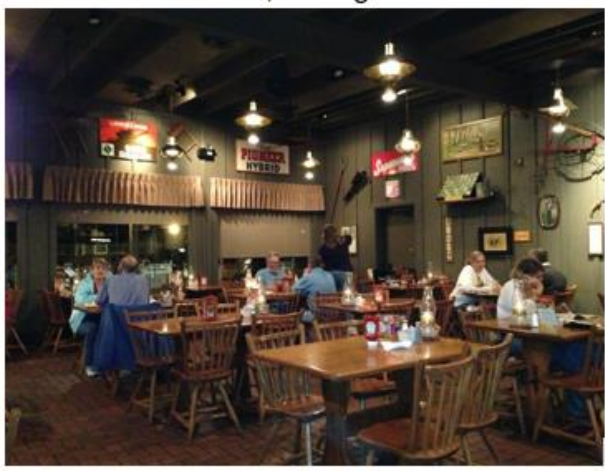

Madison, Wisconsin

Figure 2. Similarities in Cracker Barrel's Interior Design ${ }^{3}$

\footnotetext{
${ }^{3}$ Cracker Barrel restaurants are remarkably similar across the country. The upper left image shows the Milford, Connecticut store, the upper right shows the Okemos, Michigan store, the bottom left show the Stevensville, Maryland store, and the bottom right shows the Madison, Wisconsin store. Each shows the very similar wall decor, same furniture, and oil lamps. Sources (clockwise starting with Milford, CT): C., Jose. Interior. Yelp! 30 June 2015, s3-media4.fl.yelpcdn.com/bphoto/Ui32EndXjS8BeNQMLqu9vA/o.jpg; H., Richard. Interior. Yelp!, 31 Aug. 2014, s3-media3.fl.yelpcdn.com/bphoto/zHcMHZc_kYLC_bYiIWuBMw/o.jpg; L., Jessica. Interior. Yelp!, 29 July 2014, s3-media3.fl.yelpcdn.com/bphoto/EGNFrJUZOL64ggbGt6qt8A/o.jpg; M., Bill. Interior. Yelp!, 28 Sept. 2012, s3-media2.fl.yelpcdn.com/bphoto/I0wntwBNTtBzCV2VCliJEQ/o.jpg
} 
It is clear from the image analysis, and from Cracker Barrel's own website ("Bringing the Cracker Barrel," n.d), that every Cracker Barrel adheres to the same basic building design with only limited changes in seasonal decor, such as flags for the Fourth of July or fall pumpkins. Every Cracker Barrel also has a prominent, stone fireplace at the front of the restaurant with a deer head and rifle above it ("Bringing the Cracker Barrel," n.d). There are always old portraits, clocks, household items, china, or other homely artifacts on the mantle and walls devoid of any reference to a specific geographic location. Snow shoes, for instance, while not surprisingly an item featured in New Hampshire locations, were also present in South Carolina. The dining areas, too, vary very little; Figure 2 highlights this. The tables, chairs, flooring, walls, paint colors, and lamps are uniform. Patron's comments reflect this as well with one Yelp! user from New Mexico captioning their image of a table setting, "Typical Interior" (6/14/14). Another user from Arizona commented, "wouldn't be Cracker Barrel without this on the table" (9/20/16) in reference to the infamous peg game and old oil lamps on the table. It is this uniformity that contributes to a sense of placelessness within Cracker Barrel restaurants that is clearly apparent in guest images.

While the uniform nature of Cracker Barrel is a defining feature, there are minor differences that were apparent in the image analysis, most notably, the vegetation surrounding each location, the inclusion of team apparel in the general store, and a more modern design used in new restaurants. Not surprisingly, the vegetation outside the store is unique to the store's geographic location given variations in soil type and climate. The stores in Florida and South Carolina, for example, have palm trees while the Arizona stores feature cacti and the stores on the east coast have small shrubs or crepe myrtles. Placelessness, it seems, is easier to create indoors.

A second element that changes from store to store are the sports team's gear that is sold in each general store. While other elements of the "general store" remain standard, such as old fashioned candy and soda as well as home decor products, the sports apparel sold differs based on location. However, sports apparel was rarely photographed. Three states, Minnesota, Virginia, and Tennessee, are the only stores within the study where users took and posted images focused on the sport's apparel of the Vikings, Virginia Tech, and the University of Tennessee, respectively. If by no other way, it may be possible for patrons to locate themselves geographically by determining the sport's team represented in the specific Cracker Barrel. 

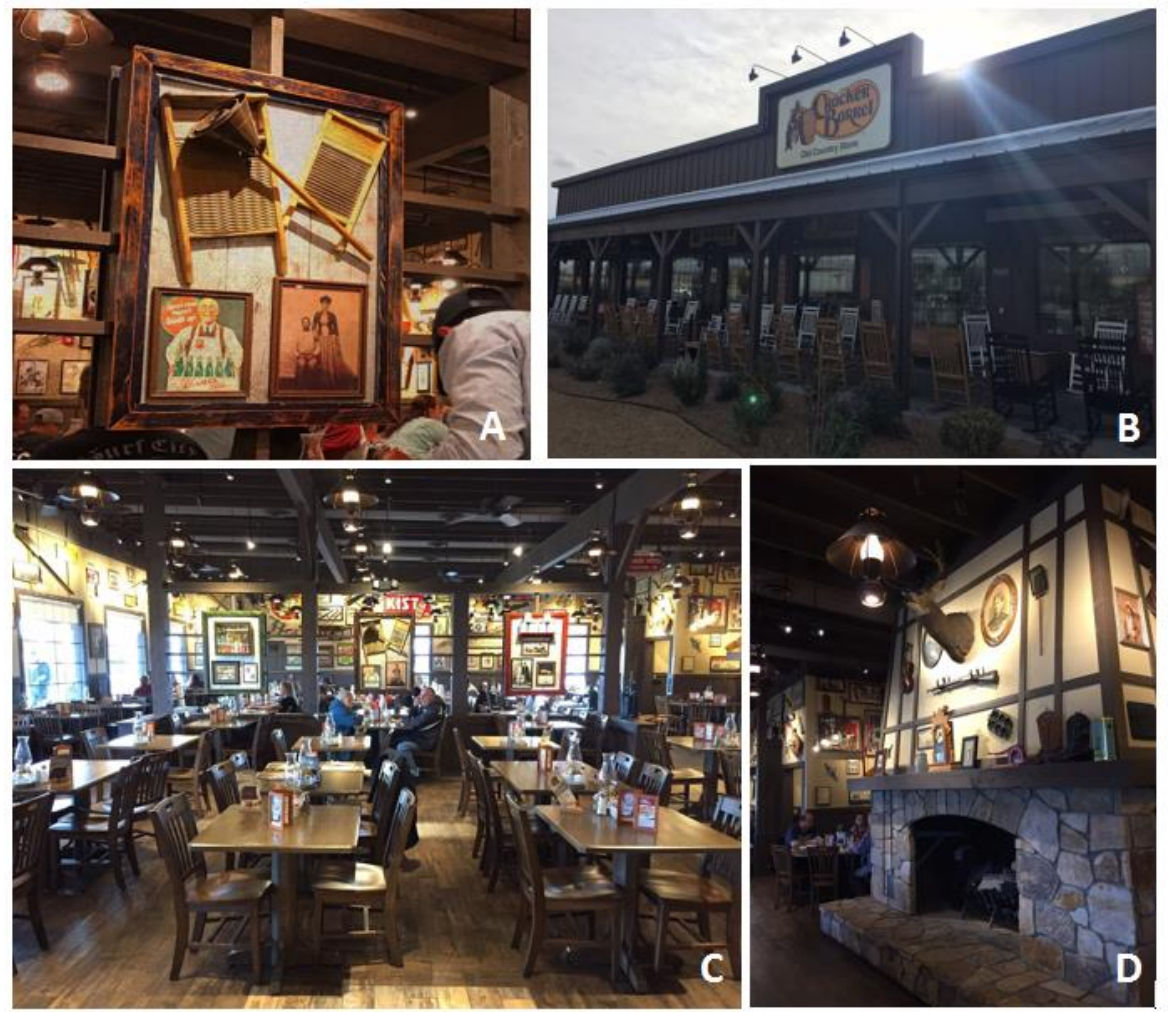

Figure 3. Las Vegas, Nevada Cracker Barrel ${ }^{4}$

A final anomaly was a new style of Cracker Barrel that is present at the newest stores. This new model closely resembles the standard and well known Cracker Barrel look, but it has a few modern twists (See Figure 3). The first store that utilizes the new model is located in Morganton, North Carolina and opened June 22, 2105 ("Cracker Barrel Opens", 2015). All fourteen stores built since then follow this new model according to follow-up image analysis using Yelp! As evident from Figure 3, the changes include a new wall color, a new way to divide the restaurant, new furniture and flooring, a slight change to the exterior of the building, and changes to the fireplace, which now extrudes from the wall out into the restaurant itself more. These changes, while quite

\footnotetext{
${ }^{4}$ These images show the new design model for new Cracker Barrels as of 2015. All of these images are from the North Las Vegas, Nevada location. (A) shows the new dividers that replaced the lattice work of the old model, (B) shows a slightly different shape to the store facade, (C) shows the restaurant overview with the new furniture and floors, and (D) shows how the fireplace is now extruded into the restaurant. Sources (A-D): A., Rob. New Model. Yelp!, 11 May 2017, s3-media1.fl.yelpcdn.com/bphoto/3kN31NetRMZQaCIbXvR51g/o.jpg; M. Michael. New Model. Yelp!, 26 Oct. 2016, s3-media4.fl.yelpcdn.com/bphoto/ZEIjZ-mawaXuCrC3xx8OAA/o.jpg; P., Jessica. New Model. Yelp!, 9 Jan. 2017, s3-media3.fl.yelpcdn.com/bphoto/ZdW_NGOKWIC986-nE7JIIw/o.jpg; P., Jessica. New Model. Yelp!, 9 Jan. 2017, s3-media1.fl.yelpcdn.com/bphoto/0gx1aKaI7RcVzdjK4oPhRA/o.jpg.
} 
obvious, are not so far removed from the original design that the store is unrecognizable. The "must haves" such as the deer head and rifle above the mantle are included in the new design.

\section{ON CREATING A SENSE OF PLACE}

Cracker Barrel is simultaneously placeless and placefull, existing on the one hand as a uniform restaurant and store that changes little based on geographic location and yet connects with visitors through nostalgic reminders of home. This relative uniformity is intentional, according to the Cracker Barrel website. Cracker Barrel was originally envisioned to fulfill an unmet need for people traveling on the ever expanding highway system ("Frequently Asked Questions," n.d). The hope was to create a place where people could "stretch their legs, refuel, eat a consistently good meal at a good price" ("Frequently Asked Questions," n.d). The founder, Dan Evins wanted to create "a place that preserved the ingredients of country life to share with travelers on the road and families from nearby" ("Frequently Asked Questions," n.d). The two key elements in this statement are the sense of place he hoped to create and his intended audience.

Further, the Cracker Barrel website notes: "Our brand is about being a home-away-from-home for everyone we welcome to our table. In some ways, it's about coming home to the charm of a simpler time and place" ("Frequently Asked Questions," n.d). It is clear that this is the sense of place Cracker Barrel works tirelessly to create. The other element in the founder's goal is to be a place for both locals and travelers. As Figure 4 shows, 518 Cracker Barrels of 637 total locations on the map are within 1.5 miles of an interstate highway for this exact reason. This creates a challenge, however, when trying to design and decorate a store that fosters personal connections with all the patrons yet also highlights the local area. The items that create a feeling of "home" vary at least to some extent across the country (Fowler \& Lipscomb, 2010, p. 107), and thus Cracker Barrel stores contain a myriad of "homey" items to appeal to both locals and travelers, including snowshoes on the wall of the North Myrtle Beach, South Carolina store. 


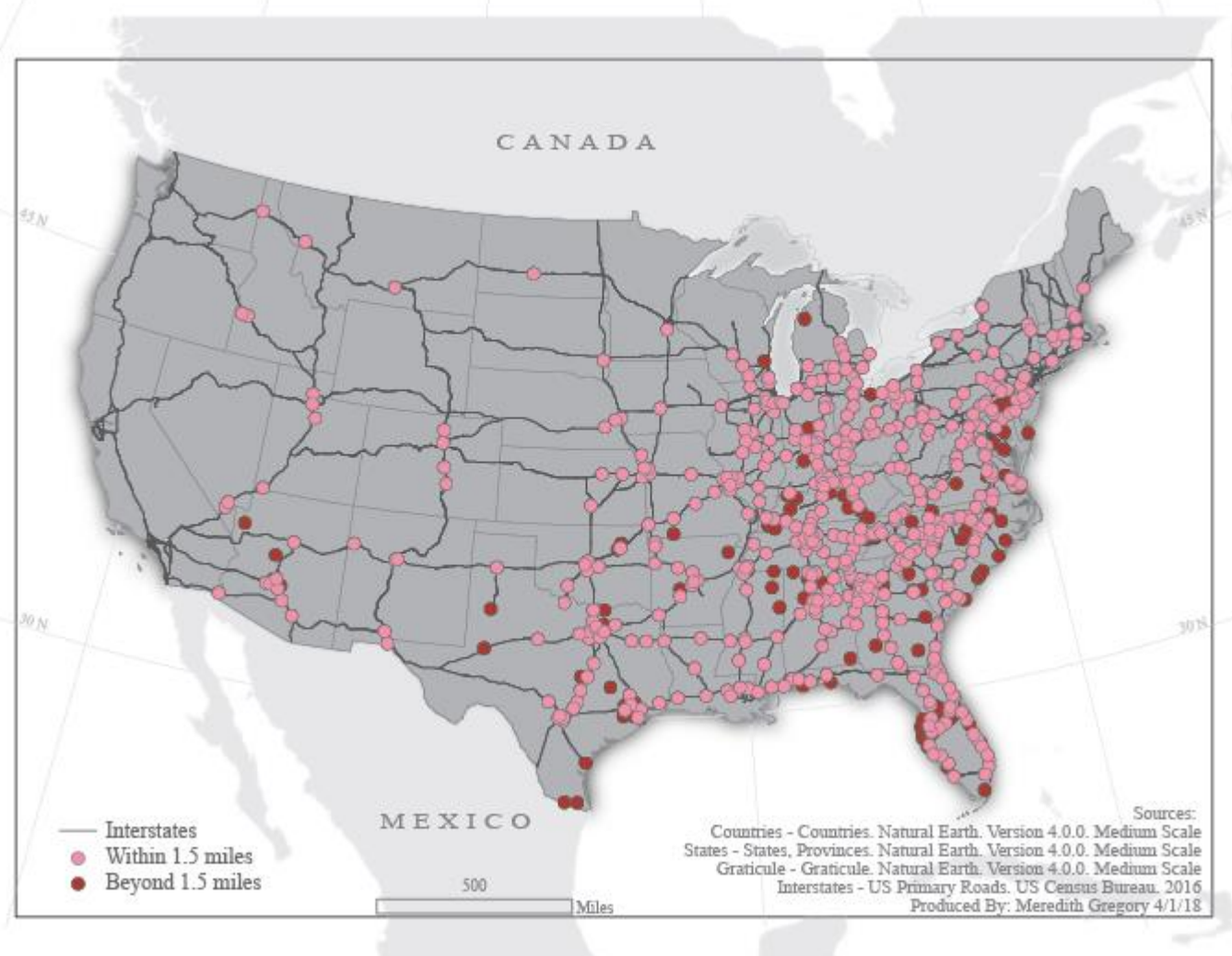

Figure 4. Map of Cracker Barrel Locations and Interstates, United States ${ }^{5}$

It is here that the tension lies between placefullness and placelessness. The Cracker Barrel website states in relation to the visitor experience, "maintaining that experience throughout our over 600 locations requires a commitment to consistency and relentless repetition" ("Frequently Asked Questions," n.d). Like any brand, Cracker Barrel wants their stores to be nationally recognizable, yet Cracker Barrel claims to also add a local twist to each store. In a news release, they state, "Each Cracker Barrel location is uniquely decorated with real American artifacts, memorabilia and signage curated by a team of experts. The walls of Cracker Barrel stores reflect the nation's rich history and by tailoring elements to the local community, offer a homespun appeal for local residents." ("Cracker Barrel Old Country," 2016). Regarding the Las Vegas, Nevada store, for example, Cracker Barrel states that "guests will see localized pieces that pay homage to the Old West, the Hoover Dam,

\footnotetext{
${ }^{5}$ This map highlights those Cracker Barrels that are directly off the primary interstates in the United States. 518 of the 637 Cracker Barrels on this map are located directly off (within 1.5 miles) interstate exits. The others are on other major secondary roads. Source: Authors' own elaboration.
} 
the circus, and recreation on Lake Mead" ("Cracker Barrel Old Country," 2016). Even after reviewing the images again, specifically looking for these items, the items that Cracker Barrel claimed to include in their attempt to "localize" the location are not identifiable. With the exception of sports gear and vegetation, the notion of local distinctiveness was not apparent in the analyzed photographs. This is not to say that local geography is completely absent from Cracker Barrel stores, but simply that visitors to Cracker Barrel did not notice, or were not prompted to post photographs, of distinctive local elements.

Although Cracker Barrel is an essentially placeless establishment in that very little changes store to store, this does not stop it from being incredibly placefull. Rather, it speaks to a tension between maintaining a national brand that is easily recognized from the side of the road and providing a consistent experience for visitors, while at the same time cultivating a distinctive sense of home and comfort that keeps these same visitors coming back. Theming plays a key role in creating this sense of both place and placelessness. Wood and Muñoz (2007, p. 243) write, "themed environments seem to flourish by giving a very expected, standardised, and controlled environment" effectively connecting theming and placelessness and providing a basis from which to work out this paradox. Theming creates both standardized experiences and placefullness thus proving that it is not a one or the other - placelessness or placefullness - situation, but that it is the interaction between them that has provided Cracker Barrel with so much success.

For Cracker Barrel's customers, the themed experience begins immediately when stepping onto the large front porch. The porch shape is an iconic design often seen in "Old West" towns and the rocking chairs are a quintessential element of a rural America. While these elements, along with many other features included in the Cracker Barrel design, may not be historically accurate of country stores, Muñoz and Wood (2009, p. 270) highlight that, "when selecting design atmospheric and aesthetic cues, reality engineers rely heavily on socially constructed, yet often inaccurate themes". Along with rocking chairs, the front porches are adorned with old product signs, farm equipment, and barrels. Each Cracker Barrel has a checkers set and a wooden church pew bench as well. All of these elements work together to create the sense of place that is Cracker Barrel.

Within the general store and restaurant, again theming is used throughout to generate a distinct sense of time and place. Every Cracker Barrel, for instance, has various artifacts hanging from the ceiling 
including old Radio Flyer red wagons, wagon wheels, old buckets, shovels and sledge hammers, and parts of a horse harness, just to name a few. The four major elements of the restaurant that create a sense of place are the artifacts on the walls, the fireplace, the table settings, and the food. The fireplace in every store is positioned just through the entrance to the restaurant from the "general store." It is a focal point of both the restaurant and Yelp! user comments as 21 images had comments about the fireplace. While this number seems low it is one of the top subjects within the comments. Cracker Barrel not only has them for aesthetics but they use it as a supplementary heat source in the winter. Each table has an old oil lamp and the infamous peg game, with one visitor commenting on a picture of the table setting: "Old timer oil lamp (my grandma had these around the house growing up)," revealing not only the generation who used these but also how this particular patron connected to the decor. Finally, the menu is the same at every location across the country and features items such as Country Fried Steak, Mama's Pancake Breakfast, and Chicken $n^{\prime}$ Dumplins. The combination of all of these signs together point back and reference this specific time and place in history. If there is any item that someone might consider representative in any way of rural 19th century America, it is highly likely to be hanging on the walls of a Cracker Barrel. In fact, 219 images of the 746 total include the wall decor to some extent. Not every Cracker Barrel has the same set of artifacts, but what variation does exist is minimal. None of these artifacts alone represent all of rural 19th century America, but together create a strong sense of place.

With such a large variety of objects, Cracker Barrel's hope is that no matter who walks through the doors, there is at least one if not many, many artifacts that they have a deeper connection with. Cracker Barrel uses these artifacts and other design choices together to create a very overt sense of rural 19th century America. Other than "Cracker Barrel" the next most used word in the comments section of these photos was "country." This alone is proof that Cracker Barrel is successful at creating this particular sense of place. Other comments such as, "Just like down South in my hometown, North Carolina!" on an image taken at the North Las Vegas, Nevada Cracker Barrel and "Nostalgic place, nice country down to earth place. Just like Mom makes it" both put this sense of place into words. While one key way to create a place is through the physical elements that construct it, it is the "ability [of the physical features] to translate an external phenomenon and link it to internal experience or 
cultural beliefs" (DeLyser, 1999, p. 608) that forms these autobiographical connections that keep visitors coming back.

The appearance and aesthetics of Cracker Barrel are carefully cultivated. Cracker Barrel owns a warehouse where "reality engineers" curate and store artifacts from across the country so that when another Cracker Barrel opens, they can package up the desired sense of place and ship it off to wherever the new store is located ("Bringing the Cracker Barrel," n.d). Not only are the aesthetics standardized but the experience is as well. Crackers Barrel acknowledges this on their website, "Our success ultimately depends on our store employees providing excellent food, friendly service and quality merchandise in a warm and inviting atmosphere - all on a daily basis" ("Frequently Asked Questions," n.d). This formula of standardization to experience, convenience in location, and speed of service, has served Cracker Barrel well in its almost 50-year history.

Studies on theming are often an assessment of authenticity (Wood \& Muñoz, 2007; Muñoz \& Wood, 2009). This study, instead, uses theming to understand how Cracker Barrel creates the sense of place that is so present in each store. If places are the features of a location that differentiate that space from another as well as the emotional connections people have with a space, then successful theming is essentially an attempt to engineer the former - the features of a location - in the hopes that an emotional connection will follow. Cracker Barrel relies on the specifically selected artifacts and other visual elements of the stores to elicit a feeling of "homeiness." Creating a sense of home is a continual and ever evolving process, however (Fowler \& Lipscomb, 2010; Phillips et al., 2011). So how then - and why - does Cracker Barrel create a "home-awayfrom-home" when it seems almost impossible, as everyone's sense of home is different and ever evolving. Cracker Barrel uses their uniformity and placelessness in their favor because once a patron has been to one Cracker Barrel they have essentially been to all of them. Consistency is not the only requirement to engineer a sense of home, however. Theming and the careful selection of artifacts reinforce a patron's nostalgic feelings and help visitors label their feelings of consistency and nostalgia as "home." Cracker Barrel includes memorabilia and decorative items from all areas of life, including fishing poles, irons, and portraits, hoping everyone who walks through the door will connect to at least one of these items as reminiscent of something from their parents' or grandparents' homes. Cracker Barrel also relies on the socially constructed theme of country living as being naturally homey so that even those who have never, for 
example, left the heart of New York City might walk into a Cracker Barrel and feel at home. Thus, even without local flair, Cracker Barrel is still able to consistently create a sense of place.

\section{CONCLUSION}

Aesthetics is the primary actor in physical place-making; the place must have something distinct about it so its identity is obvious to those interacting with it. Those involved with creating themed places arguably understand this better than anyone else. With themed locations, it is the ethnic art, decor, music, external façade, name, and various stereotyped signals' that are used to create a sense of place. Successful theming, and thus placefull places, relies on not just one of these but all of these working together. Cracker Barrel, as revealed by the image analysis, does just this, creating a place with the look and feel of a 19th century country store.

Cracker Barrel's meticulous use of artifacts to craft the placefullness that overflows from the stores, creates such a strong sense of place that even their shortcomings in incorporating local culture, which leads to the essential placelessness of the brand, do not in any way detract from the sense of place visitors feel as soon as they pull into the parking lot. Placelessness, an attribute often used to describe the unremarkable strip malls and office parks of the world, in this case is the exact feature that creates the ultimate placefullness that travelers and locals alike come in waves to experience because it is this standardization of experiences that creates reliability, familiarity, and ease - all elements of a happy home.

\section{REFERENCES}

Bringing the Cracker Barrel Look to Life (n.d.). Cracker Barrel. Retrieved February 6, 2019, from https://crackerbarrel.com/about/bringing-the-cracker-barrel-look-to-life

Cracker Barrel Old Country Store ${ }^{\circledR}$ Opens New Store in North Las Vegas, Nevada. (2016). Cracker Barrel. Retrieved April 2, 2018, from https://crackerbarrel.com/newsroom/news-releases/2016/oct/north-las-vegasopening

Cracker Barrel Opens New Store in Morganton. (2015). Cracker Barrel. Retrieved April 2, 2018, from https://crackerbarrel.com/newsroom/news-releases/2015/jul/crackerbarrel-opens-new-store-in-morganton

Crang, M. (2005). Analysing qualitative materials. In R. Flowerdew \& D. Martin (Eds.), Methods in Human Geography: A Guide for Students Doing a Research Project (pp. 218-232). New York: Pearson Education Limited.

Csurgó, B., \& Megyesi, B. (2016). The role of small towns in local place making. European Countryside, 8(4), 427-443. https://doi.org/10.1515/euco-2016-0029 
Cuba, L., \& Hummon, D. M. (1993). Constructing a sense of home: place affiliation and migration across the life cycle. Sociological Forum, 8(4), 547-572. https://doi.org/10.1007/BF01115211.

DeLyser, D. (1999). Authenticity on the ground: Engaging the past in a California ghost town. Annals of the Association of American Geographers, 89(4), 602-632.

DeBres, K., \& Sowers, J. (2009). The emergence of standardized, idealized, and placeless landscapes in Midwestern main street postcards. The Professional Geographer, 61(2), 216-230. https://doi.org/10.1080/00330120902736062.

Echtner, C. M. (1999). The semiotic paradigm: Implications for tourism research. Tourism management, 20(1), 47-57.

Frequently Asked Questions (n.d.). Cracker Barrel. Retrieved April 2, 2018, from https://crackerbarrel.com/connect/faqs.

Fowler, A. R., \& Lipscomb, C. A. (2010). Building a sense of home in rented spaces. International Journal of Housing Markets and Analysis, 3(2), 100-118.

Goss, J. (1992). Modernity and postmodernity in the retail landscape. In K. Anderson \& F. Gale (Eds.), Inventing Places: Studies in Cultural Geography (pp. 159-177). Melbourne: Longman Cheshire Pty Limited.

Harner, J., \& Kinder, F. (2011). Placelessness in a deregulated city: University Village in Colorado Springs. Urban Geography, 32(5), 730-755. https://doi.org/10.2747/02723638.32.5.730

Hough, M. (1990). Out of Place; Restoring Identity to the Regional Landscape. West Hanover, Massachusetts: Yale University Press.

Korusiewicz, M. (2015). Places in placelessness - notes on the aesthetic and strategies of place-making. Argument Biannual Philosophical Journal, 5(February), 399-413.

Knudsen, D. C., \& Rickly-Boyd, J. M. (2012). Tourism sites as semiotic signs: A critique. Annals of Tourism Research, 39(2), 1252-1254.

Lau, R. W. (2011). Tourist sights as semiotic signs: A critical commentary. Annals of Tourism Research, 38(2), 711-714.

Lau, R. W. (2014). Semiotics, objectivism and tourism: An anti-critique. Annals of Tourism Research, 44, 283-284.

Mehmetoglu, M., \& Dann, G. (2002). Atlas/ti and content/semiotic analysis in tourism research. Tourism Analysis, 8(1), 1-13.

Muñoz, C. L., \& Wood, N. T. (2009). A recipe for success: Understanding regional perceptions of authenticity in themed restaurants. International Journal of Culture, Tourism, and Hospitality Research, 3(3), 269-280. https://doi.org/10.1108/17506180910980564.

Nelson, V. (2017). An Introduction to the Geography of Tourism. London: Rowman \& Littlefield.

Pennington, J. W., \& Thomsen, R. C. (2010). A semiotic model of destination representations applied to cultural and heritage tourism marketing. Scandinavian Journal of Hospitality and Tourism, 10(1), 33-53.

Phillips, J., Walford, N., \& Hockey, A. (2011). How do unfamiliar environments convey meaning to older people? Urban dimensions of placelessness and attachment. International Journal of Ageing and Later Life, 6(2), 73-102.

Relph, E. (1976). Place and Placelessness. London: Pion.

Relph, E. (1981). Rational Landscapes and Humanistic Geography. Totowa: Barnes and Noble Books.

Rose, G. (2016). Visual Methodologies: An Introduction to the Interpretation of Visual Materials. London: Sage Publications. 
Saussure, F. (1998). Nature of the linguistic sign. In D. H. Richter (Ed.), The Critical Tradition: Classic Texts and Contemporary Trends (pp. 832-835). Boston: Bedford/St. Martin's Press.

Shao, Y., \& Lui, B. (2017). Place attachment assessment system in contemporary $\begin{array}{llll}\text { urbanism. } & \text { Procedia }\end{array}$ https://doi.org/10.1016/j.proeng.2017.07.079.

Song, C. M., \& Jeon, H. Y. (2018). A semiotic study of regional branding reflected in the slogans of Korean regions. Social Semiotics, 28(2), 230-256.

Tuan, Y. (1977). Space and Place: The Perspective of Experience. Minneapolis: University of Minnesota Press.

Williamson, J. (1978). Decoding Advertisements: Ideology and Meaning in Advertising. London: Marion Boyers.

Wood, N. T., \& Muñoz, C. L. (2007). 'No rules, just right' or is it? The role of themed restaurants as cultural ambassadors. Tourism and Hospitality Research, 7(3/4), 242255. https://doi.org/10.1057/palgrave.thr.6050047.

Zelinsky, W. (2011). Not yet a Placeless Land: Tracking an Evolving American Geography. Amherst and Boston: University of Massachusetts.

Zimmerbauer, K. (2011). From image to identity: Building regions by place promotion.
European
Planning
Studies,
19(2),
243-260.

https://doi.org/10.1080/09654313.2011.532667. 